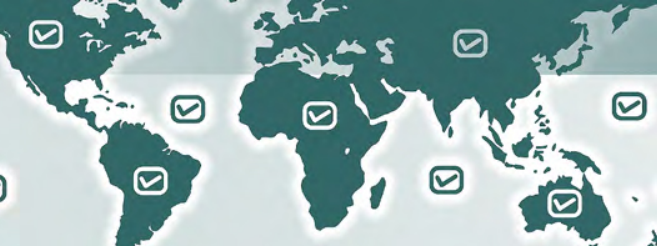

NOTES ON GEOGRAPHIC DISTRIBUTION

\title{
The Great Plains Ratsnake, Pantherophis emoryi Baird \& Girard, 1853, (Squamata: Colubridae), a new state record from Aguascalientes, México
}

\author{
Gustavo Ernesto Quintero-Díaz ${ }^{1,3}$, Armando Cardona-Arceo $^{3}$ and Rubén Alonso Carbajal-Márquez ${ }^{2,3 *}$ \\ 1 Universidad Autónoma de Aguascalientes, Centro de Ciencias Básicas, Departamento de Biología. C. P. 20131, Aguascalientes, \\ Aguascalientes, México \\ 2 El Colegio de la Frontera Sur. Departamento de Conservación de la Biodiversidad. Unidad Chetumal, Av. Centenario Km 5.5, 77014, \\ Chetumal, Quintana Roo, México \\ 3 Conservación de la Biodiversidad del Centro de México, A. C. Andador Torre de Marfil No. 100, C. P. 20229, Aguascalientes, \\ Aguascalientes, México \\ * Corresponding Author. E-mail: redman031@hotmail.com
}

\begin{abstract}
We report an adult male specimen of the snake Pantherophis emoryi from xerophytic scrub habitat in the Municipality of Real de Asientos, Aguascalientes, México. This specimen represents the first state record of P. emoryi from Aguascalientes and extends the known distribution of the species $107 \mathrm{~km}$ in a straight line west-northwest from the nearest previous record. Our specimen was found in habitat that is under heavy agricultural pressure, and recently, threatened by mining. This may indicate that other Chihuahuan Desert taxa may be found in this area.
\end{abstract}

Key words: Real de Asientos; new record; xerophytic scrub; Chihuahuan Desert

The Great Plains Ratsnake (Pantherophis emoryi Baird \& Girard, 1853) has a wide distribution; the range extends from southwestern Illinois, Missouri, southern South Dakota, and southeastern Colorado in the U.S.A., southward to San Luis Potosi and Veracruz in México (Hammerson 2007). In Mexico the range includes the states of Chihuahua, Coahuila, Durango, Hidalgo, Nuevo León, Querétaro, San Luis Potosí, Tamaulipas and Veracruz (Hammerson 2007; Lemos-Espinal and Smith 2009; Ramírez-Bautista et al. 2014; Lemos-Espinal and Smith 2015). It has been reported from Jalisco, but this is erroneous, based on misidentified juvenile specimens of Senticolis triaspis Cope, 1866 (Cruz-Sáenz et al. 2008; Flores-Cobarrubias et al. 2012). The species is listed by the IUCN as Least Concern (LC) and not listed by Norma Oficial Mexicana (NOM-059-SEMARNAT-2010) (Hammerson 2007; Diario Oficial de la Federación 2010).
At the present time, 67 species of reptiles occur in Aguascalientes, with many recently documented (Vázquez-Díaz and Quintero-Díaz 2005; Quintero-Díaz et al. 2008; Carbajal-Márquez et al. 2011; Arenas-Monroy et al. 2014; Quintero Díaz et al. 2014; Carbajal-Márquez et al. 2015). We present data on an additional species, Pantherophis emoryi, which represents a new state record.

During a field trip on 5 May 2015, with the purpose of updating the inventory of herpetofauna from Aguascalientes, ACA found a dead-on-road specimen of Pantherophis emoryi on a paved road surrounded by xerophytic scrub in the Municipality of Real de Asientos, Aguascalientes, $\left(22.284455^{\circ} \mathrm{N}, 102.067940^{\circ} \mathrm{W}\right.$ [WGS84]; 2,064 $\mathrm{m}$ above sea level). The specimen was collected under permit SEMARNAT SGPA/DGVS/05143/14, identified by J. Jesús Sigala-Rodríguez, and deposited in the Vertebrate Collection at Universidad Autónoma de Aguascalientes (voucher UAA-CZUAA-REP-0508). This specimen is an adult male (snout vent length $=840 \mathrm{~mm}$, total length $=967 \mathrm{~mm}$ ). Vegetation in the xerophytic scrub was Twisted Acacia (Acacia schaffneri), Catclaw Mimosa (Mimosa monacistra), Leatherstem (Jatropha dioica), Silver Prairie-clover (Dalea bicolor), and Prickly Pear (Opuntia spp.) (Rosales-Carrillo 2008).

Pantherophis emoryi is a medium sized snake reaching $1,530 \mathrm{~mm}$ in total length (Lemos-Espinal and Smith 2009). The head scales are: preoculars $1-1$, postoculars $2-2$, anterior temporals $2-2$ or more, supralabials $8-8$, and infralabials usually $11-13$. Midbody scale rows usually 27 but often 29, ventrals 201-236, subcaudals 60-83. Dorsal scales usually have 2 apical pits. The dorsal coloration is light gray, olive or brown, with 


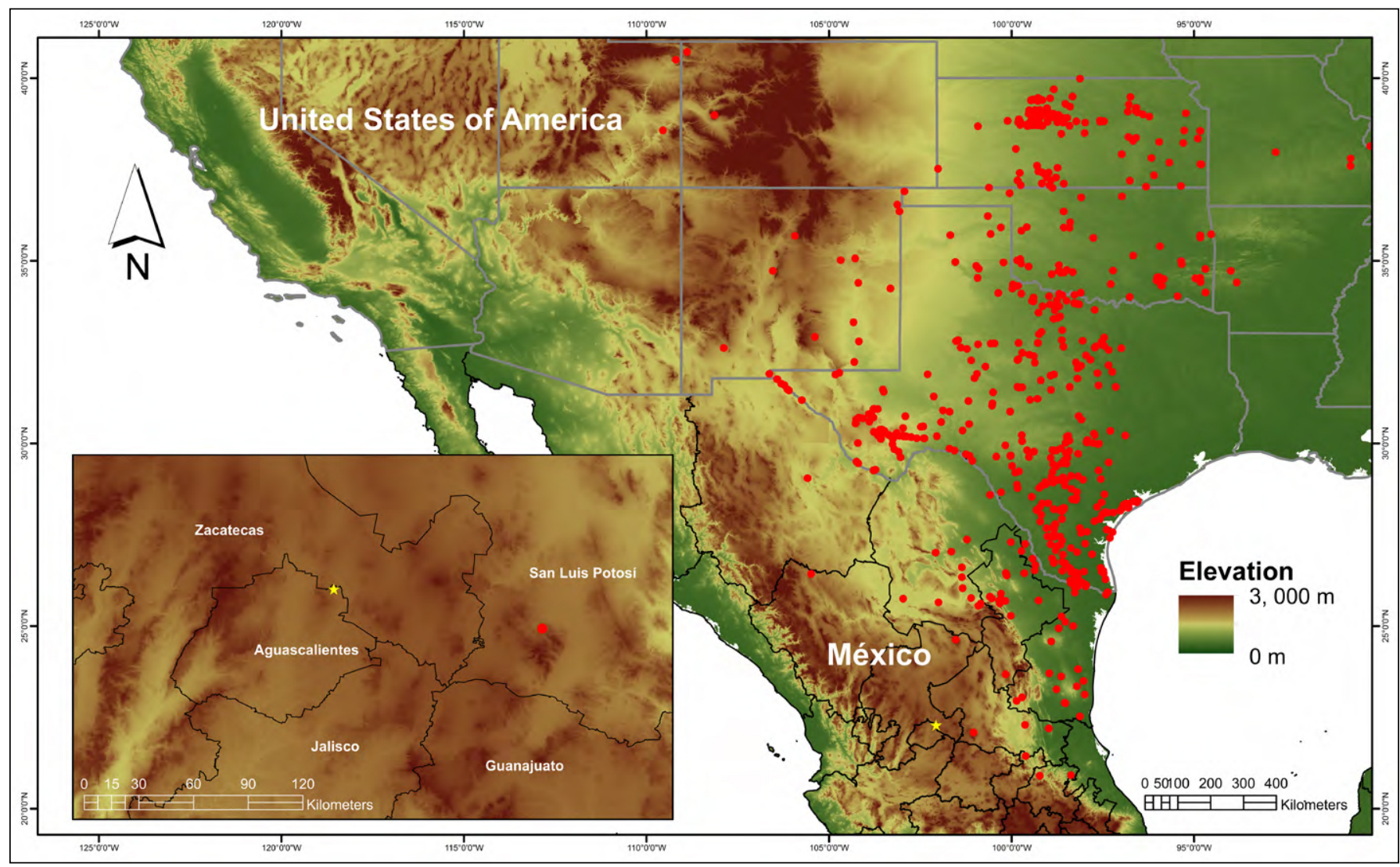

Figure 1. Collecting localities for Pantherophis emoryi across its geographic range in México and United States of America (Appendix, Table A1). The star represents the new record of Pantherophis emoryi for Aguascalientes at Real de Asientos. The black lines represent state boundaries in Mexico, and gray lines state boundaries in U.S.A.

28-45 (37) squarish or rectangular brown dorsal blotches on the body, 11-23 (16) on the tail, and a lateral series of smaller blotches. The underside of the head and much of the ventral surface is white. Small black spots are scattered posteriorly on the ventral scales, and continue on the subcaudals. The only other similar colubrid snake occurring in Aguascalientes are juveniles of Senticolis triaspis (Green Rat Snake), which differs from P. emoryi in having 31-35 dorsal scales at midbody, 80 or more dorsal blotches on the body, dark lines on the head fragmented, except for the interorbital bar, and restricted to the southwest portion of Aguascalientes; P. emoryi has 27-29 dorsal scales at midbody, 28-45 dorsal blotches, large arrow-shaped dark marks on top and sides of the head, and is restricted to northeastern Aguascalientes. No subspecies are recognized for Pantherophis emoryi (Price 1991; Burbrink 2002; LemosEspinal and Smith 2009).

This specimen represents the first record of the species for the state of Aguascalientes and extends the known geographic range $107 \mathrm{~km}$ in a straight line west-northwest from $4.8 \mathrm{~km}$ west of San Luis Potosi city, San Luis Potosi (Lemos-Espinal and Dixon 2013), and $263 \mathrm{~km}$ south-southwest from Mazapil, Zacatecas (J. Jesús Sigala-Rodríguez, pers. comm.) (Figure 1). Our specimen was found in xerophytic scrub, a habitat that in Real de Asientos is rapidly being transformed into crop and livestock production, and also threatened by mining. This and other recent herpetofaunal findings in the arid zone of Aguascalientes suggest that additional species typical of the Chihuahuan Desert may be found in this area.

\section{ACKNOWLEDGEMENTS}

We thank J. Jesus Sigala Rodríguez for comments on previous draft of this note and for information provided.

\section{LITERATURE CITED}

Arenas-Monroy, J.C., U.O. García-Vázquez, R.A. Carbajal-Márquez and A. Cardona-Arceo. 2014. A new state record for Aguascalientes, México: Phrynosoma cornutum (Squamata: Phrynosomatidae), the Texas horned Lizard. Herpetology Notes 7: 551-553. http://biotaxa.org/hn/article/view/7915/10037

Burbrink, F.T. 2002. Phylogeographic analysis of the cornsnake (Elaphe guttata) complex as inferred from maximum likelihood and Bayesian analyses. Molecular Phylogenetics and Evolution 25(3): 465-476. doi: 10.1016/S1055-7903(02)00306-8

Carbajal-Márquez, R.A., G.E. Quintero-Díaz and Z.Y. GonzálezSaucedo. 2011. Rinocheilus lecontei (Longnose Snake): Geographic distribution. Herpetological Review 42(2): 244.

Carbajal-Márquez, R.A., G.E. Quintero-Díaz and E.A. Rivas-Mercado. 2015. Crotalus basiliscus (Mexican West Coast Rattlesnake): Geographic distribution. Herpetological Review 46(2): 219.

Cruz-Sáenz, D., C.E. Gudiño-Larios, C.D. Jimeno-Sevilla, R. LópezVelázquez and J. Cortés-Aguilar. Guía de Reptiles y Anfibios de Arcediano. Gobierno de Jalisco. Comisión Estatal del Agua. México. 126 pp. 
Diario Oficial de la Federación. 2010. Norma Oficial Mexicana NOM059-SEMARNAT-2010, Protección ambiental - Especies nativas de México de flora y fauna silvestres - Categorías de riesgo y especificaciones para su inclusión, exclusión o cambio - Lista de especies en riesgo, 30 de diciembre de 2010. http://biblioteca. semarnat.gob.mx/janium/Documentos/Ciga/agenda/DOFsr/ DO2454.pdf

Flores-Cobarrubias, E., D. Cruz-Sáenz and D. Lazcano 2012. Notes on the herpetofauna of western Mexico 6: amphibians and reptiles of Hostotipaquillo, Jalisco, Mexico. Bulletin of the Chicago Herpetological Society 47(2): 21-26. http://www.chicagoherp. org/bulletin/47(2).pdf

GBIF. 2016. Global Biodiversity Information Facility. Accessed at http://www.gbif.org, 20 April 2016.

Lemos-Espinal, J.A. and H.M. Smith. 2009. Amphibians and reptiles of the state of Chihuahua, D. F., Mexico. UNAM, CONABIO, University of Colorado at Boulder. 613 pp.

Lemos-Espinal, J.A. and J.R. Dixon. 2013. Amphibians and reptiles of the state of San Luis Potosí. Eagle Mountain, Utah: Eagle Mountain Publishing. 300 pp.

Lemos-Espinal, J.A. and G.R. Smith. 2015. Amphibians and reptiles of the state of Hidalgo, Mexico. Checklist 11(3): 1642. doi: 10.15560/11.3.1642

Price, R.M. 1991. Senticolis triaspis. Catalogue of American Amphibians and Reptiles 525: 1-4.

Quintero-Díaz, G.E., J. Vázquez-Díaz and J.J. Sigala R. 2008. Capítulo 3. Biodiversidad.3.16. "Reptiles".pp.141-145, in:La Biodiversidad en Aguascalientes: Libro de Estado. Comisión Nacional para el
Conocimiento y Uso de la Biodiversidad (CONABIO), Instituto del Medio Ambiente del Estado de Aguascalientes (IMAE), Universidad Autónoma de Aguascalientes (UAA). México.

Quintero-Díaz, G.E., S. Rojas-Quezada and R.A. Carbajal-Márquez. 2014. Lampropeltis polyzona (Mexican Milksnake): Geographic distribution. Herpetological Review 45(4): 664.

Ramírez-Bautista, A., U. Hernández-Salinas, R. Cruz-Elizalde, C. Berriozabal-Islas, D. Lara-Tufiño, I. Goyenechea and J. Castillo-Cerón. 2014. Los anfibios y reptiles de Hidalgo, México: Diversidad, Biogeografía y Conservación. Universidad Autónoma del Estado de Hidalgo y Sociedad Herpetológica Mexicana A.C. $387 \mathrm{pp}$.

Rosales-Carrillo, O. 2008. Capítulo 3. Biodiversidad. 3. 3. "MATORRAL". pp. 89-91, in: La Biodiversidad en Aguascalientes: Libro de Estado. México: Comisión Nacional para el Conocimiento y Uso de la Biodiversidad (CONABIO), Instituto del Medio Ambiente del Estado de Aguascalientes (IMAE), Universidad Autónoma de Aguascalientes (UAA).

Vázquez-Díaz, D.J. and G.E. Quintero-Díaz. 2005. Anfibios y Reptiles de Aguascalientes. México: CONABIO, Centro de Investigaciones Multidisciplinarios de Aguascalientes. 318 pp.

Author contributions: RACM, GEQD and ACA collected the data, RACM and GEQD wrote the text. GEQD made the map.

Received: 5 July 2015

Accepted: 26 May 2016

Academic editor: Ross MacCulloch

\section{APPENDIX}

Table A1. Records of Pantherophis emoryi obtained from the present study, personal communications, published literature and museum specimens consulted in GBIF. AMNH: American Museum of Natural History; BYU: Brigham Young University; CAS: California Academy of Science; CM: Carnegie Museum of Natural History; FHSM: Fort Hays State University; FMNH: Field Museum of Natural History; KU: University of Kansas Natural History Museum; LACM: Natural History Museum of Los Angeles County; MCZ: Museum of Comparative Zoology; MSB: Museum of Southwestern Biology; NCSM: North Carolina Museum of Natural Sciences; OMNH: Osaka Museum of Natural History; OSUM: Ohio State University; SDNHM: San Diego Natural History Museum; TCWC: Texas Cooperative Wildlife Collection; UAA: Universidad Autónoma de Aguascalientes; UANL: Universidad Autónoma de Nuevo León; UBCBBM: University of British Columbia; UCM: University of Colorado Museum; UMMZ: University of Michigan Museum of Zoology; USNM: National Museum of Natural History, Smithsonian Institution; UTEP: University of Texas at El Paso; YPM: Yale University, Peabody Museum of Natural History.

\begin{tabular}{|c|c|c|c|c|c|c|}
\hline Country & State & Collection & Catalog Number & Latitude & Longitude & Source \\
\hline$M X$ & Aguascalientes & UAA & CZUAA-REP-0508 & 22.2845 & -102.0679 & This study \\
\hline$M X$ & Chihuahua & $\mathrm{CM}$ & 59911 & 29.05 & -105.59 & GBIF \\
\hline$M X$ & Chihuahua & $\mathrm{CM}$ & 59913 & 29.05 & -105.59 & GBIF \\
\hline$M X$ & Chihuahua & $\mathrm{CM}$ & 59912 & 29.05 & -105.59 & GBIF \\
\hline$M X$ & Chihuahua & iNaturalist & 1609908 & 29.4562 & -104.1986 & GBIF \\
\hline$M X$ & Chihuahua & iNaturalist & 1636826 & 29.2661 & -103.7848 & GBIF \\
\hline $\mathrm{MX}$ & Coahuila & $\mathrm{CM}$ & 59914 & 26.6103 & -101.3602 & GBIF \\
\hline$M X$ & Coahuila & $\mathrm{CM}$ & 42762 & 27.0103 & -102.0802 & GBIF \\
\hline$M X$ & Coahuila & $\mathrm{CM}$ & 59916 & 25.7503 & -102.9702 & GBIF \\
\hline$M X$ & Coahuila & $\mathrm{CM}$ & 48184 & 27.0403 & -101.6502 & GBIF \\
\hline$M X$ & Coahuila & $\mathrm{CM}$ & 59915 & 27.3703 & -101.2202 & GBIF \\
\hline$M X$ & Coahuila & iNaturalist & 719828 & 25.6153 & -100.846 & GBIF \\
\hline$M X$ & Coahuila & iNaturalist & 1930054 & 28.5928 & -100.5875 & GBIF \\
\hline$M X$ & Coahuila & SDNHM & 60475 & 26.04 & -101.34 & GBIF \\
\hline$M X$ & Coahuila & UTEP & H-3797 & 25.6503 & -102.0002 & GBIF \\
\hline$M X$ & Coahuila & UTEP & H-6336 & 25.7703 & -101.1102 & GBIF \\
\hline$M X$ & Durango & CAS & 135101 & 26.4303 & -105.4903 & GBIF \\
\hline$M X$ & Durango & CAS & 87410 & 26.4351 & -105.4902 & GBIF \\
\hline$M X$ & Hidalgo & - & - & 20.9032 & -099.2257 & Ramírez-Bautista et al. 2014 \\
\hline$M X$ & Hidalgo & - & - & 20.9193 & -098.3725 & Ramírez-Bautista et al. 2014 \\
\hline$M X$ & Nuevo León & $\mathrm{CM}$ & 66486 & 25.6703 & -100.2802 & GBIF \\
\hline
\end{tabular}


Table A1. Continued.

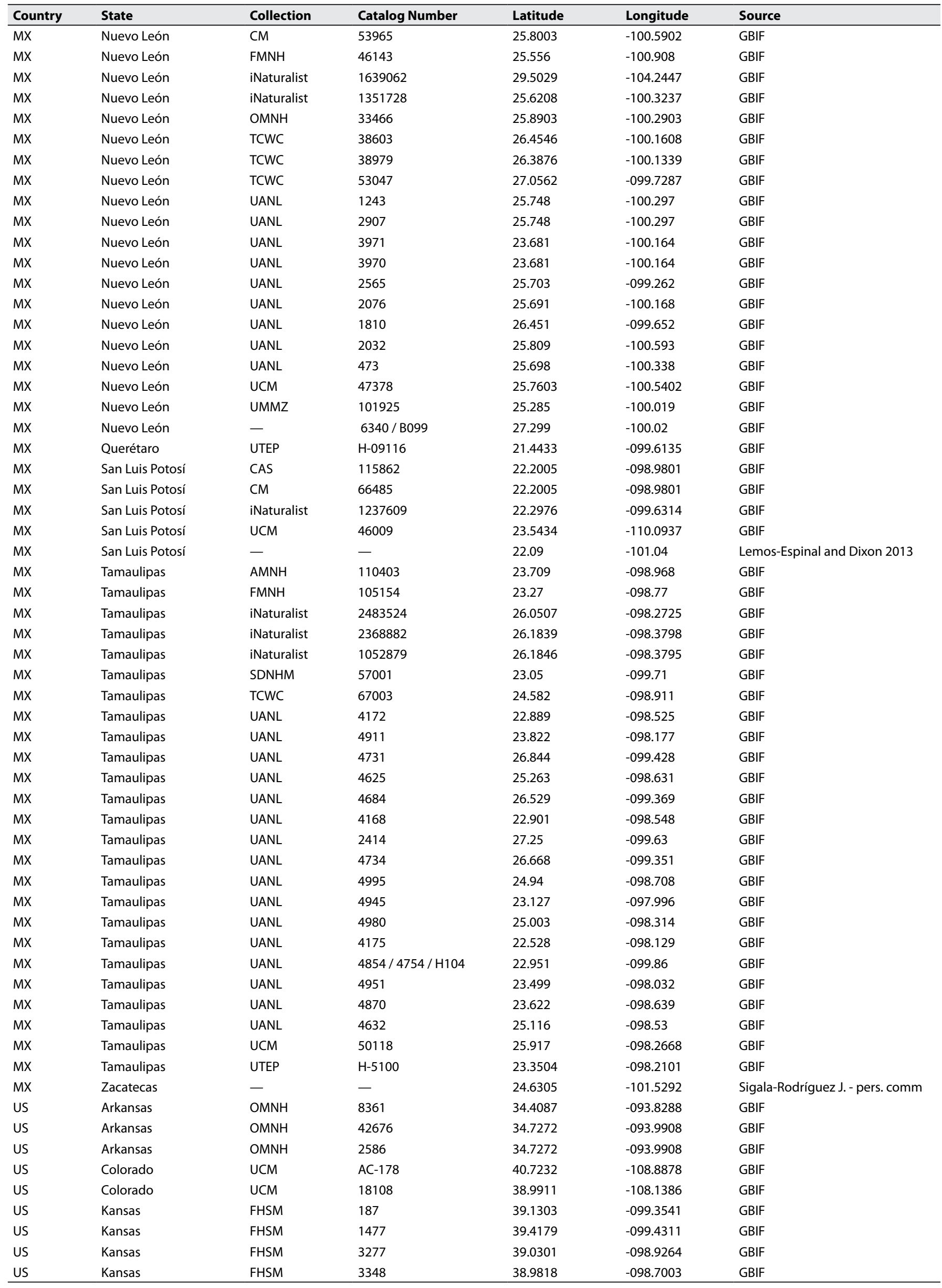


Table A1. Continued.

\begin{tabular}{|c|c|c|c|c|c|c|}
\hline Country & State & Collection & Catalog Number & Latitude & Longitude & Source \\
\hline US & Kansas & FHSM & 3375 & 38.999 & -098.925 & GBIF \\
\hline US & Kansas & FHSM & 3376 & 38.9818 & -098.7003 & GBIF \\
\hline US & Kansas & FHSM & 3549 & 38.8738 & -098.7579 & GBIF \\
\hline US & Kansas & FHSM & 3694 & 38.6929 & -099.5665 & GBIF \\
\hline US & Kansas & FHSM & 4302 & 38.9862 & -098.9635 & GBIF \\
\hline US & Kansas & FHSM & 4304 & 38.9862 & -098.9635 & GBIF \\
\hline US & Kansas & FHSM & 4464 & 38.8205 & -098.2254 & GBIF \\
\hline US & Kansas & FHSM & 6739 & 38.9727 & -098.8731 & GBIF \\
\hline US & Kansas & MCZ & R-29034 & 39.2767 & -096.7884 & GBIF \\
\hline US & Kansas & FHSM & 12770 & 37.3414 & -096.0981 & GBIF \\
\hline US & Kansas & FHSM & 13346 & 38.7519 & -099.338 & GBIF \\
\hline US & Kansas & FHSM & 13954 & 38.7854 & -098.5841 & GBIF \\
\hline US & Kansas & UTEP & H-5115 & 38.7858 & -097.9838 & GBIF \\
\hline US & Kansas & FHSM & 15946 & 39.0413 & -095.2283 & GBIF \\
\hline US & Kansas & FHSM & 4301 & 38.9422 & -098.9086 & GBIF \\
\hline US & Kansas & FHSM & 4514 & 37.5458 & -098.7397 & GBIF \\
\hline US & Kansas & FHSM & 6000 & 38.839 & -097.5822 & GBIF \\
\hline US & Kansas & FHSM & 11567 & 39.4535 & -098.9439 & GBIF \\
\hline US & Kansas & FHSM & 11594 & 38.7128 & -099.4323 & GBIF \\
\hline US & Kansas & FHSM & 12092 & 37.4072 & -099.7766 & GBIF \\
\hline US & Kansas & FHSM & 12883 & 37.6102 & -099.3029 & GBIF \\
\hline US & Kansas & FHSM & 1395 & 38.8579 & -099.4258 & GBIF \\
\hline US & Kansas & FHSM & 1398 & 38.8579 & -099.4258 & GBIF \\
\hline US & Kansas & FHSM & 1431 & 37.1965 & -096.7592 & GBIF \\
\hline US & Kansas & FHSM & 3572 & 38.9428 & -099.2153 & GBIF \\
\hline US & Kansas & FHSM & 4299 & 38.8727 & -098.7373 & GBIF \\
\hline US & Kansas & FHSM & 6109 & 38.7459 & -099.5498 & GBIF \\
\hline US & Kansas & FHSM & 9070 & 39.4155 & -099.2766 & GBIF \\
\hline US & Kansas & FHSM & 12903 & 38.9011 & -098.5715 & GBIF \\
\hline US & Kansas & FHSM & 3380 & 38.9498 & -098.8617 & GBIF \\
\hline US & Kansas & FHSM & 3396 & 38.9027 & -098.7165 & GBIF \\
\hline US & Kansas & FHSM & 3467 & 39.0301 & -098.9264 & GBIF \\
\hline US & Kansas & FHSM & 11789 & 38.9984 & -098.8727 & GBIF \\
\hline US & Kansas & $\mathrm{CM}$ & S5434 & 38.56 & -094.8301 & GBIF \\
\hline US & Kansas & CM & S8671 & 38.57 & -095.2701 & GBIF \\
\hline US & Kansas & UTEP & Ho-16 & 38.7743 & -099.7475 & GBIF \\
\hline US & Kansas & FHSM & 457 & 38.8528 & -099.2965 & GBIF \\
\hline US & Kansas & FHSM & 5863 & 37.4561 & -099.2491 & GBIF \\
\hline US & Kansas & FHSM & 6110 & 38.7459 & -099.5498 & GBIF \\
\hline US & Kansas & FHSM & 7908 & 38.6972 & -099.156 & GBIF \\
\hline US & Kansas & FHSM & 11294 & 37.0008 & -098.8779 & GBIF \\
\hline US & Kansas & FHSM & 11596 & 38.7128 & -099.4323 & GBIF \\
\hline US & Kansas & $\mathrm{KU}$ & 221199 & 37.0323 & -096.3176 & GBIF \\
\hline US & Kansas & FHSM & 2270 & 38.8423 & -098.6194 & GBIF \\
\hline US & Kansas & FHSM & 2378 & 38.8706 & -099.3622 & GBIF \\
\hline US & Kansas & FHSM & 3418 & 38.7247 & -099.7631 & GBIF \\
\hline US & Kansas & FHSM & 3426 & 38.912 & -098.9842 & GBIF \\
\hline US & Kansas & FHSM & 3598 & 38.7324 & -099.3938 & GBIF \\
\hline US & Kansas & FHSM & 7883 & 39.0472 & -099.0309 & GBIF \\
\hline US & Kansas & FHSM & 12383 & 38.0655 & -099.8834 & GBIF \\
\hline US & Kansas & FHSM & 1393 & 38.9143 & -099.332 & GBIF \\
\hline US & Kansas & FHSM & 1399 & 38.9294 & -098.8638 & GBIF \\
\hline US & Kansas & FHSM & 1428 & 38.8579 & -099.4258 & GBIF \\
\hline US & Kansas & FHSM & 1479 & 39.4179 & -099.4311 & GBIF \\
\hline US & Kansas & FHSM & 2495 & 38.9615 & -098.8542 & GBIF \\
\hline US & Kansas & FHSM & 2518 & 38.9641 & -098.8514 & GBIF \\
\hline
\end{tabular}


Table A1. Continued.

\begin{tabular}{|c|c|c|c|c|c|c|}
\hline Country & State & Collection & Catalog Number & Latitude & Longitude & Source \\
\hline US & Kansas & FHSM & 7699 & 37.9214 & -096.9813 & GBIF \\
\hline US & Kansas & FHSM & 9537 & 37.2823 & -098.7799 & GBIF \\
\hline US & Kansas & FHSM & 11302 & 37.1148 & -098.5683 & GBIF \\
\hline US & Kansas & FHSM & 16345 & 39.9885 & -098.1268 & GBIF \\
\hline US & Kansas & FHSM & 6113 & 38.9365 & -098.6974 & GBIF \\
\hline US & Kansas & FHSM & 7104 & 38.9165 & -098.5876 & GBIF \\
\hline US & Kansas & FHSM & 8896 & 39.4902 & -096.743 & GBIF \\
\hline US & Kansas & MCZ & R-29035 & 39.2767 & -096.7884 & GBIF \\
\hline US & Kansas & FHSM & 15010 & 38.9145 & -099.3123 & GBIF \\
\hline US & Kansas & FHSM & 12082 & 39.1025 & -099.251 & GBIF \\
\hline US & Kansas & FHSM & 12263 & 37.448 & -099.2599 & GBIF \\
\hline US & Kansas & UTEP & H-5119 & 38.7936 & -099.8163 & GBIF \\
\hline US & Kansas & FHSM & 1397 & 38.8579 & -099.4258 & GBIF \\
\hline US & Kansas & FHSM & 4473 & 38.7104 & -099.2997 & GBIF \\
\hline US & Kansas & FHSM & 8633 & 37.3983 & -099.1322 & GBIF \\
\hline US & Kansas & FHSM & 8435 & 37.5211 & -102.0135 & GBIF \\
\hline US & Kansas & FHSM & 9584 & 37.281 & -099.0408 & GBIF \\
\hline US & Kansas & FHSM & 11009 & 38.3717 & -096.5936 & GBIF \\
\hline US & Kansas & FHSM & 11599 & 38.7128 & -099.4323 & GBIF \\
\hline US & Kansas & FHSM & 12083 & 38.9486 & -099.4189 & GBIF \\
\hline US & Kansas & FHSM & 13539 & 37.0067 & -100.6177 & GBIF \\
\hline US & Kansas & FHSM & 750 & 37.0749 & -098.9635 & GBIF \\
\hline US & Kansas & FHSM & 1482 & 38.8335 & -099.4088 & GBIF \\
\hline US & Kansas & FHSM & 4303 & 38.9862 & -098.9635 & GBIF \\
\hline US & Kansas & FHSM & 6106 & 38.7459 & -099.5498 & GBIF \\
\hline US & Kansas & FHSM & 11067 & 38.3935 & -096.8015 & GBIF \\
\hline US & Kansas & FHSM & 13955 & 38.7854 & -098.5841 & GBIF \\
\hline US & Kansas & CM & S9844 & 39.19 & -096.5801 & GBIF \\
\hline US & Kansas & FHSM & 2283 & 38.8108 & -099.7616 & GBIF \\
\hline US & Kansas & FHSM & 3417 & 38.7247 & -099.7631 & GBIF \\
\hline US & Kansas & FHSM & 9654 & 39.3764 & -099.2782 & GBIF \\
\hline US & Kansas & FHSM & 12937 & 37.417 & -098.9846 & GBIF \\
\hline US & Kansas & FHSM & 455 & 39.0436 & -099.3142 & GBIF \\
\hline US & Kansas & FHSM & 2279 & 38.7395 & -099.3545 & GBIF \\
\hline US & Kansas & FHSM & 3548 & 38.8738 & -098.7579 & GBIF \\
\hline US & Kansas & FHSM & 5366 & 38.9451 & -098.6702 & GBIF \\
\hline US & Kansas & FHSM & 7720 & 39.0441 & -098.9852 & GBIF \\
\hline US & Kansas & FHSM & 8964 & 39.2994 & -098.5274 & GBIF \\
\hline US & Kansas & FHSM & 11429 & 39.0745 & -099.0933 & GBIF \\
\hline US & Kansas & FHSM & 13953 & 38.7854 & -098.5841 & GBIF \\
\hline US & Kansas & UTEP & H-5118 & 38.478 & -098.6366 & GBIF \\
\hline US & Kansas & FHSM & 13596 & 38.2586 & -095.9564 & GBIF \\
\hline US & Kansas & FHSM & 1396 & 38.8579 & -099.4258 & GBIF \\
\hline US & Kansas & FHSM & 1478 & 39.4179 & -099.4311 & GBIF \\
\hline US & Kansas & FHSM & 2280 & 38.7395 & -099.3545 & GBIF \\
\hline US & Kansas & FHSM & 3295 & 39.4142 & -099.4202 & GBIF \\
\hline US & Kansas & FHSM & 3693 & 38.6929 & -099.5665 & GBIF \\
\hline US & Kansas & FHSM & 4372 & 39.1025 & -099.1829 & GBIF \\
\hline US & Kansas & FHSM & 12081 & 39.1025 & -099.251 & GBIF \\
\hline US & Kansas & FHSM & 12300 & 38.7992 & -099.0218 & GBIF \\
\hline US & Kansas & UTEP & H-5116 & 38.8097 & -097.5746 & GBIF \\
\hline US & Kansas & UTEP & $\mathrm{H}-5117$ & 38.9832 & -099.0766 & GBIF \\
\hline US & Kansas & FHSM & 456 & 38.8764 & -099.3716 & GBIF \\
\hline US & Kansas & FHSM & 1302 & 38.7175 & -099.2259 & GBIF \\
\hline US & Kansas & FHSM & 1429 & 38.8192 & -099.3905 & GBIF \\
\hline US & Kansas & FHSM & 1480 & 38.8341 & -099.4285 & GBIF \\
\hline
\end{tabular}


Table A1. Continued.

\begin{tabular}{|c|c|c|c|c|c|c|}
\hline Country & State & Collection & Catalog Number & Latitude & Longitude & Source \\
\hline US & Kansas & FHSM & 4374 & 39.023 & -099.5306 & GBIF \\
\hline US & Kansas & FHSM & 6740 & 38.9727 & -098.8731 & GBIF \\
\hline US & Kansas & FHSM & 7084 & 39.043 & -098.9908 & GBIF \\
\hline US & Kansas & FHSM & 11773 & 38.9178 & -099.0022 & GBIF \\
\hline US & Kansas & FHSM & 14019 & 38.8339 & -100.1078 & GBIF \\
\hline US & Kansas & FHSM & 12931 & 38.3497 & -094.8997 & GBIF \\
\hline US & Kansas & FHSM & 3160 & 38.9303 & -098.7088 & GBIF \\
\hline US & Kansas & FHSM & 5537 & 38.8232 & -097.466 & GBIF \\
\hline US & Kansas & FHSM & 7498 & 39.1256 & -099.5162 & GBIF \\
\hline US & Kansas & FHSM & 8047 & 39.1836 & -099.0283 & GBIF \\
\hline US & Kansas & FHSM & 13697 & 38.9512 & -096.2228 & GBIF \\
\hline US & Kansas & FHSM & 13952 & 38.7854 & -098.5841 & GBIF \\
\hline US & Kansas & USNM & 2259.600083 & 38.2272 & -095.2965 & GBIF \\
\hline US & Kansas & FHSM & 13541 & 37.203 & -098.9217 & GBIF \\
\hline US & Kansas & FHSM & 4370 & 38.9058 & -099.4453 & GBIF \\
\hline US & Kansas & FHSM & 4371 & 39.1321 & -099.2206 & GBIF \\
\hline US & Kansas & FHSM & 5299 & 38.6858 & -100.9195 & GBIF \\
\hline US & Kansas & FHSM & 5864 & 37.4561 & -099.2491 & GBIF \\
\hline US & Kansas & FHSM & 9748 & 39.3978 & -099.4723 & GBIF \\
\hline US & Kansas & iNaturalist & 1400496 & 39.0884 & -096.8139 & GBIF \\
\hline US & Kansas & FHSM & 12930 & 38.916 & -098.476 & GBIF \\
\hline US & Kansas & FHSM & 3522 & 39.1169 & -099.2751 & GBIF \\
\hline US & Kansas & FHSM & 3683 & 38.9371 & -098.9813 & GBIF \\
\hline US & Kansas & FHSM & 4300 & 38.8727 & -098.7373 & GBIF \\
\hline US & Kansas & FHSM & 4472 & 38.7104 & -099.2997 & GBIF \\
\hline US & Kansas & FHSM & 9576 & 37.2822 & -098.7858 & GBIF \\
\hline US & Kansas & FHSM & 10805 & 37.0521 & -095.3595 & GBIF \\
\hline US & Kansas & FHSM & 11776 & 39.0279 & -099.0017 & GBIF \\
\hline US & Kansas & FHSM & 186 & 39.1303 & -099.3541 & GBIF \\
\hline US & Kansas & FHSM & 1481 & 38.9294 & -098.8638 & GBIF \\
\hline US & Kansas & FHSM & 2574 & 38.727 & -099.7575 & GBIF \\
\hline US & Kansas & FHSM & 3377 & 38.9818 & -098.7003 & GBIF \\
\hline US & Kansas & FHSM & 3533 & 38.9428 & -099.2153 & GBIF \\
\hline US & Kansas & FHSM & 6111 & 38.724 & -099.2839 & GBIF \\
\hline US & Kansas & FHSM & 6768 & 37.1209 & -099.1706 & GBIF \\
\hline US & Kansas & FHSM & 8086 & 39.5025 & -098.3293 & GBIF \\
\hline US & Kansas & FHSM & 11829 & 39.019 & -098.8539 & GBIF \\
\hline US & Kansas & OMNH & 34313 & 38.2864 & -096.6731 & GBIF \\
\hline US & Kansas & FHSM & 12915 & 37.6452 & -094.8265 & GBIF \\
\hline US & Kansas & MCZ & $\mathrm{R}-5419$ & 39.19 & -096.5859 & GBIF \\
\hline US & Kansas & FHSM & 1403 & 38.9294 & -098.8638 & GBIF \\
\hline US & Kansas & FHSM & 2516 & 38.9641 & -098.8514 & GBIF \\
\hline US & Kansas & FHSM & 4296 & 37.0743 & -099.7476 & GBIF \\
\hline US & Kansas & FHSM & 5394 & 37.2042 & -099.8363 & GBIF \\
\hline US & Kansas & FHSM & 8039 & 38.7994 & -099.0196 & GBIF \\
\hline US & Kansas & FHSM & 11828 & 39.0308 & -098.8538 & GBIF \\
\hline US & Kansas & FHSM & 11597 & 38.7128 & -099.4323 & GBIF \\
\hline US & Kansas & FHSM & 11595 & 38.7128 & -099.4323 & GBIF \\
\hline US & Kansas & FHSM & 1430 & 38.8339 & -099.2613 & GBIF \\
\hline US & Kansas & FHSM & 1483 & 38.8571 & -099.4614 & GBIF \\
\hline US & Kansas & FHSM & 2573 & 38.727 & -099.7575 & GBIF \\
\hline US & Kansas & FHSM & 3298 & 39.4043 & -099.1568 & GBIF \\
\hline US & Kansas & FHSM & 3370 & 38.8738 & -098.7579 & GBIF \\
\hline US & Kansas & FHSM & 6107 & 38.7459 & -099.5498 & GBIF \\
\hline US & Kansas & FHSM & 6112 & 38.9963 & -098.7249 & GBIF \\
\hline
\end{tabular}


Table A1. Continued.

\begin{tabular}{|c|c|c|c|c|c|c|}
\hline Country & State & Collection & Catalog Number & Latitude & Longitude & Source \\
\hline US & Kansas & FHSM & 1394 & 38.9143 & -099.332 & GBIF \\
\hline US & Kansas & FHSM & 2517 & 38.9641 & -098.8514 & GBIF \\
\hline US & Kansas & FHSM & 3425 & 38.8713 & -098.7355 & GBIF \\
\hline US & Kansas & FHSM & 4369 & 38.8491 & -099.4281 & GBIF \\
\hline US & Kansas & FHSM & 6108 & 38.7459 & -099.5498 & GBIF \\
\hline US & Kansas & FHSM & 8040 & 38.7994 & -099.0196 & GBIF \\
\hline US & Kansas & FHSM & 11602 & 38.7128 & -099.4323 & GBIF \\
\hline US & Kansas & FHSM & 11598 & 38.7128 & -099.4323 & GBIF \\
\hline US & Kansas & FHSM & 12382 & 38.0655 & -099.8834 & GBIF \\
\hline US & Kansas & iNaturalist & 459674 & 39.1045 & -096.5955 & GBIF \\
\hline US & Kansas & $\mathrm{KU}$ & 337079 & 39.1178 & -096.5818 & GBIF \\
\hline US & Kansas & FHSM & 2269 & 39.0797 & -099.2453 & GBIF \\
\hline US & Kansas & FHSM & 3297 & 39.4043 & -099.1568 & GBIF \\
\hline US & Kansas & FHSM & 4373 & 38.7279 & -099.3222 & GBIF \\
\hline US & Kansas & FHSM & 8434 & 37.5211 & -102.0135 & GBIF \\
\hline US & Kansas & FHSM & 9749 & 39.0637 & -096.5489 & GBIF \\
\hline US & Kansas & FHSM & 11068 & 38.3935 & -096.8015 & GBIF \\
\hline US & Kansas & FHSM & 11399 & 37.5236 & -102.012 & GBIF \\
\hline US & Kansas & $\mathrm{OMNH}$ & 37947 & 39.2233 & -098.397 & GBIF \\
\hline US & Kansas & FHSM & 756 & 38.8758 & -099.7975 & GBIF \\
\hline US & Kansas & FHSM & 3471 & 38.9355 & -098.798 & GBIF \\
\hline US & Kansas & FHSM & 4375 & 38.7175 & -099.2259 & GBIF \\
\hline US & Kansas & FHSM & 4465 & 38.6994 & -098.4107 & GBIF \\
\hline US & Kansas & FHSM & 5536 & 38.8232 & -097.466 & GBIF \\
\hline US & Kansas & FHSM & 8062 & 37.6917 & -095.6767 & GBIF \\
\hline US & Kansas & FHSM & 11066 & 38.3935 & -096.8015 & GBIF \\
\hline US & Kansas & FHSM & 11601 & 38.7128 & -099.4323 & GBIF \\
\hline US & Kansas & OSUM & 607 & 38.5 & -098 & GBIF \\
\hline US & Kansas & FHSM & 4298 & 38.8727 & -098.7373 & GBIF \\
\hline US & Kansas & FHSM & 6261 & 37.6439 & -094.8006 & GBIF \\
\hline US & Kansas & FHSM & 7508 & 38.7942 & -098.7445 & GBIF \\
\hline US & Kansas & FHSM & 9662 & 39.0089 & -096.4015 & GBIF \\
\hline US & Kansas & FHSM & 13029 & 37.6397 & -094.806 & GBIF \\
\hline US & Misuri & iNaturalist & 67922 & 37.802 & -090.7099 & GBIF \\
\hline US & Misuri & iNaturalist & 388151 & 38.1446 & -090.1682 & GBIF \\
\hline US & Misuri & iNaturalist & 127953 & 37.5975 & -090.7133 & GBIF \\
\hline US & Misuri & iNaturalist & 388857 & 37.9778 & -092.7647 & GBIF \\
\hline US & New Mexico & MSB & 72005 & 35.02 & -104.68 & GBIF \\
\hline US & New Mexico & MSB & 72545 & 34.73 & -106.54 & GBIF \\
\hline US & New Mexico & CAS & 71794 & 35.6869 & -105.9372 & GBIF \\
\hline US & New Mexico & MSB & 71883 & 36.54 & -103.13 & GBIF \\
\hline US & New Mexico & iNaturalist & 83816 & 34.2445 & -103.3178 & GBIF \\
\hline US & New Mexico & iNaturalist & 345563 & 33.3186 & -104.33 & GBIF \\
\hline US & New Mexico & TCWC & 95057 & 32.7968 & -104.1828 & GBIF \\
\hline US & New Mexico & MSB & 72006 & 32.92 & -105.39 & GBIF \\
\hline US & New Mexico & FHSM & 12598 & 32.2334 & -104.3067 & GBIF \\
\hline US & New Mexico & $\mathrm{KU}$ & 289015 & 36.357 & -103.0717 & GBIF \\
\hline US & New Mexico & MSB & 65757 & 34.4 & -104.2 & GBIF \\
\hline US & New Mexico & LACM & 2321 & 35.0683 & -104.2819 & GBIF \\
\hline US & New Mexico & UTEP & H-19520 & 32.6149 & -107.8817 & GBIF \\
\hline US & Oklahoma & OMNH & 10289 & 35.1468 & -096.67 & GBIF \\
\hline US & Oklahoma & OMNH & 12901 & 34.7553 & -098.4885 & GBIF \\
\hline US & Oklahoma & $\mathrm{OMNH}$ & 30880 & 34.0141 & -096.7615 & GBIF \\
\hline US & Oklahoma & $\mathrm{OMNH}$ & 22890 & 36.3541 & -098.5722 & GBIF \\
\hline US & Oklahoma & OMNH & 25291 & 36.7379 & -098.0921 & GBIF \\
\hline
\end{tabular}


Table A1. Continued.

\begin{tabular}{|c|c|c|c|c|c|c|}
\hline Country & State & Collection & Catalog Number & Latitude & Longitude & Source \\
\hline US & Oklahoma & OMNH & 5080 & 34.4203 & -094.8242 & GBIF \\
\hline US & Oklahoma & $\mathrm{OMNH}$ & 33993 & 36.9041 & -102.935 & GBIF \\
\hline US & Oklahoma & OMNH & 35825 & 34.777 & -094.6911 & GBIF \\
\hline US & Oklahoma & OMNH & 42941 & 34.4555 & -095.9715 & GBIF \\
\hline US & Oklahoma & $\mathrm{OMNH}$ & 39264 & 35.7289 & -094.533 & GBIF \\
\hline US & Oklahoma & OMNH & 41876 & 34.3298 & -095.9053 & GBIF \\
\hline US & Oklahoma & OMNH & 8213 & 34.712 & -098.6794 & GBIF \\
\hline US & Oklahoma & OMNH & 5081 & 34.524 & -094.9689 & GBIF \\
\hline US & Oklahoma & OMNH & 6142 & 35.6651 & -094.8319 & GBIF \\
\hline US & Oklahoma & OMNH & 8860 & 34.7358 & -097.2207 & GBIF \\
\hline US & Oklahoma & OMNH & 13187 & 34.8851 & -098.7035 & GBIF \\
\hline US & Oklahoma & OMNH & 13742 & 36.8518 & -100.0555 & GBIF \\
\hline US & Oklahoma & $\mathrm{OMNH}$ & 25976 & 36.7672 & -096.977 & GBIF \\
\hline US & Oklahoma & OMNH & 40100 & 35.6751 & -094.832 & GBIF \\
\hline US & Oklahoma & OMNH & 42943 & 34.5494 & -096.0114 & GBIF \\
\hline US & Oklahoma & $\mathrm{OMNH}$ & 42940 & 34.458 & -096.0232 & GBIF \\
\hline US & Oklahoma & OMNH & 41875 & 34.3167 & -095.884 & GBIF \\
\hline US & Oklahoma & OMNH & 43943 & 34.0315 & -095.4536 & GBIF \\
\hline US & Oklahoma & OMNH & 6140 & 35.638 & -094.8289 & GBIF \\
\hline US & Oklahoma & $\mathrm{OMNH}$ & 6523 & 34.3298 & -095.9053 & GBIF \\
\hline US & Oklahoma & OMNH & 5079 & 34.5538 & -095.9649 & GBIF \\
\hline US & Oklahoma & OMNH & 29266 & 35.909 & -098.4111 & GBIF \\
\hline US & Oklahoma & $\mathrm{OMNH}$ & 35640 & 34.6869 & -098.5556 & GBIF \\
\hline US & Oklahoma & SDNHM & 56014 & 36.0557 & -098.3925 & GBIF \\
\hline US & Oklahoma & OMNH & 12900 & 34.7553 & -098.4885 & GBIF \\
\hline US & Oklahoma & $\mathrm{OMNH}$ & 11160 & 34.9129 & -095.3406 & GBIF \\
\hline US & Oklahoma & OMNH & 26868 & 35.3999 & -095.9364 & GBIF \\
\hline US & Oklahoma & OMNH & 27691 & 34.1368 & -094.6874 & GBIF \\
\hline US & Oklahoma & OMNH & 6141 & 35.7002 & -094.8294 & GBIF \\
\hline US & Oklahoma & OMNH & 5073 & 34.4555 & -095.9715 & GBIF \\
\hline US & Oklahoma & OMNH & 5077 & 34.4599 & -095.9603 & GBIF \\
\hline US & Oklahoma & OMNH & 7066 & 35.9253 & -099.5848 & GBIF \\
\hline US & Oklahoma & OMNH & 42939 & 34.5067 & -095.8311 & GBIF \\
\hline US & Oklahoma & OMNH & 5076 & 34.5494 & -096.0114 & GBIF \\
\hline US & Oklahoma & OMNH & 26889 & 34.7168 & -098.7075 & GBIF \\
\hline US & Oklahoma & OMNH & 42736 & 35.9253 & -099.5848 & GBIF \\
\hline US & Oklahoma & OMNH & 29278 & 34.9295 & -099.7339 & GBIF \\
\hline US & Oklahoma & OMNH & 41951 & 35.6281 & -097.7619 & GBIF \\
\hline US & Oklahoma & OMNH & 43820 & 34.5361 & -094.8296 & GBIF \\
\hline US & Oklahoma & OMNH & 5078 & 34.5572 & -095.9595 & GBIF \\
\hline US & Oklahoma & OMNH & 6555 & 34.3167 & -095.884 & GBIF \\
\hline US & Oklahoma & $\mathrm{OMNH}$ & 9223 & 34.0315 & -095.4536 & GBIF \\
\hline US & Oklahoma & OMNH & 9224 & 35.0155 & -099.8457 & GBIF \\
\hline US & Oklahoma & OMNH & 35636 & 34.6773 & -098.6497 & GBIF \\
\hline US & Oklahoma & OMNH & 35826 & 34.78 & -094.693 & GBIF \\
\hline US & Oklahoma & OMNH & 42945 & 34.4203 & -094.8242 & GBIF \\
\hline US & Oklahoma & OMNH & 35637 & 34.693 & -098.3239 & GBIF \\
\hline US & Oklahoma & OMNH & 4448 & 34.638 & -098.8976 & GBIF \\
\hline US & Oklahoma & OMNH & 8137 & 34.712 & -098.6794 & GBIF \\
\hline US & Oklahoma & OMNH & 25825 & 34.845 & -099.5322 & GBIF \\
\hline US & Oklahoma & OMNH & 26438 & 35.826 & -099.73 & GBIF \\
\hline US & Oklahoma & OMNH & 40264 & 34.3655 & -097.2843 & GBIF \\
\hline US & Oklahoma & $\mathrm{OMNH}$ & 41877 & 35.0504 & -099.7651 & GBIF \\
\hline US & Oklahoma & OMNH & 8360 & 34.9922 & -095.3552 & GBIF \\
\hline US & Oklahoma & OMNH & 43944 & 35.0155 & -099.8457 & GBIF \\
\hline US & Oklahoma & UTEP & $\mathrm{H}-8454$ & 35.9122 & -098.5697 & GBIF \\
\hline US & Oklahoma & OMNH & 5074 & 34.5067 & -095.8311 & GBIF \\
\hline US & Oklahoma & OMNH & 5075 & 34.4614 & -095.9884 & GBIF \\
\hline US & Oklahoma & OMNH & 5072 & 34.458 & -096.0232 & GBIF \\
\hline US & Texas & iNaturalist & 830798 & 30.6998 & -104.1164 & GBIF \\
\hline US & Texas & iNaturalist & 1482802 & 29.2635 & -097.6186 & GBIF \\
\hline
\end{tabular}


Table A1. Continued.

\begin{tabular}{|c|c|c|c|c|c|c|}
\hline Country & State & Collection & Catalog Number & Latitude & Longitude & Source \\
\hline US & Texas & iNaturalist & 1568967 & 29.2837 & -103.7282 & GBIF \\
\hline US & Texas & TCWC & 14988 & 34.9696 & -101.54 & GBIF \\
\hline US & Texas & TCWC & 33626 & 26.2505 & -098.3896 & GBIF \\
\hline US & Texas & TCWC & 61541 & 35.7055 & -101.6776 & GBIF \\
\hline US & Texas & TCWC & 67488 & 32.4319 & -099.5164 & GBIF \\
\hline US & Texas & TCWC & 79757 & 32.741 & -100.4688 & GBIF \\
\hline US & Texas & TCWC & 82468 & 28.2021 & -098.8364 & GBIF \\
\hline US & Texas & UTEP & $\mathrm{H}-16230$ & 27.4589 & -099.0214 & GBIF \\
\hline US & Texas & iNaturalist & 786691 & 30.1954 & -102.9543 & GBIF \\
\hline US & Texas & iNaturalist & 796050 & 28.2157 & -098.622 & GBIF \\
\hline US & Texas & iNaturalist & 1106787 & 30.7148 & -103.7825 & GBIF \\
\hline US & Texas & TCWC & 38239 & 26.1047 & -098.2459 & GBIF \\
\hline US & Texas & TCWC & 38776 & 28.9473 & -099.0818 & GBIF \\
\hline US & Texas & TCWC & 38853 & 33.6597 & -097.7207 & GBIF \\
\hline US & Texas & TCWC & 60487 & 32.2143 & -099.3618 & GBIF \\
\hline US & Texas & TCWC & 64618 & 28.7755 & -099.8367 & GBIF \\
\hline US & Texas & TCWC & 65077 & 32.755 & -099.2768 & GBIF \\
\hline US & Texas & TCWC & 77184 & 33.6442 & -098.8175 & GBIF \\
\hline US & Texas & TCWC & 79950 & 29.815 & -099.5758 & GBIF \\
\hline US & Texas & iNaturalist & 1627260 & 32.6268 & -097.7252 & GBIF \\
\hline US & Texas & TCWC & 38854 & 32.9913 & -099.2491 & GBIF \\
\hline US & Texas & TCWC & 77170 & 33.9085 & -098.4916 & GBIF \\
\hline US & Texas & TCWC & 77171 & 26.2225 & -098.1948 & GBIF \\
\hline US & Texas & TCWC & 79248 & 27.932 & -098.6186 & GBIF \\
\hline US & Texas & TCWC & 79254 & 27.1933 & -098.7417 & GBIF \\
\hline US & Texas & FHSM & 14948 & 30.2127 & -103.0619 & GBIF \\
\hline US & Texas & iNaturalist & 1379768 & 27.8641 & -099.2152 & GBIF \\
\hline US & Texas & UTEP & H-13784 & 31.7614 & -106.4186 & GBIF \\
\hline US & Texas & iNaturalist & 1058270 & 28.2667 & -099.2206 & GBIF \\
\hline US & Texas & iNaturalist & 1580712 & 30.1401 & -102.3878 & GBIF \\
\hline US & Texas & iNaturalist & 1608416 & 28.3561 & -096.8679 & GBIF \\
\hline US & Texas & iNaturalist & 1590110 & 32.7809 & -097.5344 & GBIF \\
\hline US & Texas & iNaturalist & 1628219 & 30.8063 & -103.9088 & GBIF \\
\hline US & Texas & iNaturalist & 1621815 & 30.5884 & -101.9283 & GBIF \\
\hline US & Texas & iNaturalist & 1472950 & 27.6755 & -099.0434 & GBIF \\
\hline US & Texas & iNaturalist & 1472926 & 26.9526 & -098.5939 & GBIF \\
\hline US & Texas & iNaturalist & 1472916 & 26.601 & -098.4217 & GBIF \\
\hline US & Texas & iNaturalist & 1517578 & 28.2841 & -096.8216 & GBIF \\
\hline US & Texas & iNaturalist & 449713 & 30.0283 & -097.9237 & GBIF \\
\hline US & Texas & iNaturalist & 674036 & 30.2298 & -097.7212 & GBIF \\
\hline US & Texas & iNaturalist & 743998 & 26.4467 & -097.4779 & GBIF \\
\hline US & Texas & iNaturalist & 741701 & 31.4022 & -100.5066 & GBIF \\
\hline US & Texas & iNaturalist & 1949613 & 28.3053 & -096.8053 & GBIF \\
\hline US & Texas & iNaturalist & 278751 & 27.6875 & -098.1308 & GBIF \\
\hline US & Texas & iNaturalist & 1703539 & 30.5476 & -104.2694 & GBIF \\
\hline US & Texas & iNaturalist & 1771738 & 30.3823 & -103.5511 & GBIF \\
\hline US & Texas & iNaturalist & 1270908 & 26.3707 & -098.2266 & GBIF \\
\hline US & Texas & SDNHM & 57041 & 30.2341 & -103.5663 & GBIF \\
\hline US & Texas & CAS & 74427 & 29.4466 & -098.494 & GBIF \\
\hline US & Texas & TCWC & 93868 & 27.4179 & -097.3034 & GBIF \\
\hline US & Texas & TCWC & 39280 & 28.1781 & -099.3157 & GBIF \\
\hline US & Texas & TCWC & 64013 & 28.7837 & -099.245 & GBIF \\
\hline US & Texas & TCWC & 65075 & 32.755 & -099.2768 & GBIF \\
\hline US & Texas & TCWC & 77186 & 33.8095 & -098.9462 & GBIF \\
\hline US & Texas & TCWC & 77191 & 34.0961 & -098.6946 & GBIF \\
\hline US & Texas & TCWC & 95145 & 25.8674 & -097.4125 & GBIF \\
\hline US & Texas & TCWC & 93013 & 29.4831 & -097.3411 & GBIF \\
\hline US & Texas & TCWC & 58401 & 32.2936 & -097.8384 & GBIF \\
\hline US & Texas & TCWC & 72478 & 34.3556 & -099.9634 & GBIF \\
\hline US & Texas & TCWC & 77168 & 34.3172 & -099.8243 & GBIF \\
\hline US & Texas & TCWC & 77172 & 33.9085 & -098.4916 & GBIF \\
\hline
\end{tabular}


Table A1. Continued.

\begin{tabular}{|c|c|c|c|c|c|c|}
\hline Country & State & Collection & Catalog Number & Latitude & Longitude & Source \\
\hline US & Texas & TCWC & 77178 & 33.8172 & -098.1953 & GBIF \\
\hline US & Texas & TCWC & 79240 & 29.5317 & -098.5683 & GBIF \\
\hline US & Texas & TCWC & 82466 & 29.0281 & -097.555 & GBIF \\
\hline US & Texas & UTEP & $H-3562$ & 31.9089 & -106.6335 & GBIF \\
\hline US & Texas & UTEP & H-18592 & 31.1874 & -105.7443 & GBIF \\
\hline US & Texas & iNaturalist & 776912 & 28.9525 & -098.5685 & GBIF \\
\hline US & Texas & TCWC & 31093 & 28.2621 & -097.6541 & GBIF \\
\hline US & Texas & TCWC & 38980 & 29.0768 & -098.594 & GBIF \\
\hline US & Texas & TCWC & 52025 & 28.6075 & -097.4596 & GBIF \\
\hline US & Texas & TCWC & 52847 & 26.1218 & -098.1963 & GBIF \\
\hline US & Texas & TCWC & 71159 & 32.2773 & -101.1079 & GBIF \\
\hline US & Texas & TCWC & 72479 & 34.9572 & -100.2214 & GBIF \\
\hline US & Texas & TCWC & 77165 & 33.7581 & -098.6953 & GBIF \\
\hline US & Texas & UTEP & H-09989 & 31.7629 & -106.4244 & GBIF \\
\hline US & Texas & UTEP & H-17492 & 31.7633 & -106.4228 & GBIF \\
\hline US & Texas & iNaturalist & 760506 & 30.213 & -103.0617 & GBIF \\
\hline US & Texas & iNaturalist & 906160 & 26.4778 & -098.3692 & GBIF \\
\hline US & Texas & iNaturalist & 704585 & 29.6611 & -100.0352 & GBIF \\
\hline US & Texas & iNaturalist & 1107216 & 29.6305 & -099.5222 & GBIF \\
\hline US & Texas & iNaturalist & 1334323 & 29.0823 & -098.9104 & GBIF \\
\hline US & Texas & iNaturalist & 1409852 & 32.5905 & -101.2225 & GBIF \\
\hline US & Texas & iNaturalist & 1545748 & 28.3625 & -098.9978 & GBIF \\
\hline US & Texas & iNaturalist & 1622300 & 30.6567 & -104.2565 & GBIF \\
\hline US & Texas & iNaturalist & 1627297 & 30.8019 & -103.8489 & GBIF \\
\hline US & Texas & iNaturalist & 1594301 & 28.2382 & -096.8048 & GBIF \\
\hline US & Texas & iNaturalist & 1707276 & 27.2567 & -097.9859 & GBIF \\
\hline US & Texas & iNaturalist & 1644884 & 33.9955 & -099.4002 & GBIF \\
\hline US & Texas & iNaturalist & 1930484 & 28.2517 & -096.7913 & GBIF \\
\hline US & Texas & iNaturalist & 1849221 & 30.1911 & -102.8822 & GBIF \\
\hline US & Texas & iNaturalist & 1921964 & 26.7237 & -098.5692 & GBIF \\
\hline US & Texas & iNaturalist & 496939 & 29.0481 & -098.8802 & GBIF \\
\hline US & Texas & iNaturalist & 693280 & 26.7166 & -098.5249 & GBIF \\
\hline US & Texas & iNaturalist & 681319 & 32.8299 & -101.4623 & GBIF \\
\hline US & Texas & iNaturalist & 879037 & 29.7365 & -098.5129 & GBIF \\
\hline US & Texas & YPM & YPM HERR 002789 & 32.6731 & -097.4603 & GBIF \\
\hline US & Texas & YPM & YPM HERR 002722 & 30.9442 & -103.6667 & GBIF \\
\hline US & Texas & CAS & 74478 & 31.5492 & -097.1464 & GBIF \\
\hline US & Texas & TCWC & 5219 & 35.9149 & -100.2955 & GBIF \\
\hline US & Texas & TCWC & 38774 & 27.795 & -098.7978 & GBIF \\
\hline US & Texas & TCWC & 64616 & 28.8721 & -099.8524 & GBIF \\
\hline US & Texas & TCWC & 77174 & 33.9085 & -098.4916 & GBIF \\
\hline US & Texas & TCWC & 77192 & 33.757 & -099.1499 & GBIF \\
\hline US & Texas & UTEP & $\mathrm{H}-18305$ & 28.6756 & -098.2143 & GBIF \\
\hline US & Texas & iNaturalist & 1137489 & 27.7078 & -098.8373 & GBIF \\
\hline US & Texas & TCWC & 38236 & 30.7492 & -098.1577 & GBIF \\
\hline US & Texas & UTEP & H-17974 & 31.8859 & -104.8221 & GBIF \\
\hline US & Texas & UTEP & H-20785 & 30.9513 & -103.7738 & GBIF \\
\hline US & Texas & TCWC & 18313 & 26.551 & -098.372 & GBIF \\
\hline US & Texas & TCWC & 20313 & 34.0293 & -098.9715 & GBIF \\
\hline US & Texas & TCWC & 20407 & 32.9007 & -097.4656 & GBIF \\
\hline US & Texas & TCWC & 33618 & 26.9678 & -097.7947 & GBIF \\
\hline US & Texas & TCWC & 65274 & 32.0574 & -098.1895 & GBIF \\
\hline US & Texas & TCWC & 77180 & 33.5848 & -099.2615 & GBIF \\
\hline US & Texas & TCWC & 85492 & 34.1206 & -100.3647 & GBIF \\
\hline US & Texas & TCWC & 90408 & 32.7511 & -099.8073 & GBIF \\
\hline US & Texas & iNaturalist & 1462855 & 27.6813 & -099.0413 & GBIF \\
\hline US & Texas & UTEP & H-18944 & 33.103 & -098.6134 & GBIF \\
\hline US & Texas & UBCBBM & H001164 & 28.0959 & -097.4636 & GBIF \\
\hline US & Texas & iNaturalist & 429756 & 29.2291 & -098.8748 & GBIF \\
\hline US & Texas & iNaturalist & 706734 & 32.61 & -096.9925 & GBIF \\
\hline US & Texas & iNaturalist & 714684 & 29.0083 & -098.0453 & GBIF \\
\hline
\end{tabular}


Table A1. Continued.

\begin{tabular}{|c|c|c|c|c|c|c|}
\hline Country & State & Collection & Catalog Number & Latitude & Longitude & Source \\
\hline US & Texas & iNaturalist & 644963 & 27.5393 & -098.8418 & GBIF \\
\hline US & Texas & iNaturalist & 652666 & 33.4366 & -098.7371 & GBIF \\
\hline US & Texas & iNaturalist & 758584 & 28.3237 & -096.9166 & GBIF \\
\hline US & Texas & iNaturalist & 808684 & 30.3822 & -103.6003 & GBIF \\
\hline US & Texas & iNaturalist & 859814 & 33.9393 & -099.4142 & GBIF \\
\hline US & Texas & iNaturalist & 1490930 & 30.2214 & -103.3335 & GBIF \\
\hline US & Texas & iNaturalist & 1791857 & 30.2108 & -102.9862 & GBIF \\
\hline US & Texas & iNaturalist & 1754597 & 30.208 & -103.196 & GBIF \\
\hline US & Texas & iNaturalist & 1878569 & 30.6898 & -104.0767 & GBIF \\
\hline US & Texas & iNaturalist & 2118478 & 28.3101 & -096.8018 & GBIF \\
\hline US & Texas & iNaturalist & 2478965 & 32.2842 & -099.8379 & GBIF \\
\hline US & Texas & iNaturalist & 2478848 & 32.3093 & -099.816 & GBIF \\
\hline US & Texas & iNaturalist & 247588 & 31.8913 & -098.8398 & GBIF \\
\hline US & Texas & YPM & YPM HERR 002719 & 30.6 & -103.8942 & GBIF \\
\hline US & Texas & iNaturalist & 1578799 & 31.1013 & -100.5241 & GBIF \\
\hline US & Texas & iNaturalist & 1740720 & 30.2038 & -102.9566 & GBIF \\
\hline US & Texas & iNaturalist & 1692448 & 28.3615 & -099.1584 & GBIF \\
\hline US & Texas & iNaturalist & 2543492 & 28.5169 & -098.2245 & GBIF \\
\hline US & Texas & iNaturalist & 350347 & 28.6663 & -100.2887 & GBIF \\
\hline US & Texas & iNaturalist & 776911 & 28.979 & -098.5752 & GBIF \\
\hline US & Texas & iNaturalist & 786627 & 30.1745 & -102.7467 & GBIF \\
\hline US & Texas & iNaturalist & 1137620 & 29.8186 & -098.8813 & GBIF \\
\hline US & Texas & TCWC & 91827 & 29.9814 & -103.2656 & GBIF \\
\hline US & Texas & TCWC & 14864 & 31.5897 & -097.6186 & GBIF \\
\hline US & Texas & TCWC & 52442 & 29.374 & -098.7751 & GBIF \\
\hline US & Texas & TCWC & 63025 & 31.79 & -101.0143 & GBIF \\
\hline US & Texas & TCWC & 64012 & 28.8423 & -099.1725 & GBIF \\
\hline US & Texas & TCWC & 68359 & 28.3895 & -097.5333 & GBIF \\
\hline US & Texas & UTEP & H-19752 & 30.3426 & -103.4802 & GBIF \\
\hline US & Texas & UTEP & H-19805 & 30.219 & -096.8839 & GBIF \\
\hline US & Texas & TCWC & 94408 & 29.6696 & -100.0209 & GBIF \\
\hline US & Texas & TCWC & 61525 & 34.5335 & -100.9291 & GBIF \\
\hline US & Texas & TCWC & 68731 & 31.5511 & -099.8389 & GBIF \\
\hline US & Texas & TCWC & 77181 & 33.5848 & -099.2615 & GBIF \\
\hline US & Texas & TCWC & 77185 & 33.768 & -098.7975 & GBIF \\
\hline US & Texas & TCWC & 79251 & 27.4537 & -098.6711 & GBIF \\
\hline US & Texas & TCWC & 79996 & 28.2022 & -098.8483 & GBIF \\
\hline US & Texas & TCWC & 82047 & 29.374 & -098.7751 & GBIF \\
\hline US & Texas & TCWC & 87095 & 32.8109 & -098.0548 & GBIF \\
\hline US & Texas & TCWC & 87096 & 32.8109 & -098.0548 & GBIF \\
\hline US & Texas & MCZ & R-6822 & 31.4151 & -103.5007 & GBIF \\
\hline US & Texas & UTEP & $\mathrm{H}-124$ & 31.5978 & -106.2238 & GBIF \\
\hline US & Texas & UTEP & $\mathrm{H}-14715$ & 30.9055 & -101.8803 & GBIF \\
\hline US & Texas & UTEP & H-15432 & 30.213 & -103.0755 & GBIF \\
\hline US & Texas & iNaturalist & 858120 & 29.8157 & -098.7587 & GBIF \\
\hline US & Texas & TCWC & 64818 & 28.7576 & -098.3581 & GBIF \\
\hline US & Texas & TCWC & 67072 & 28.855 & -098.5397 & GBIF \\
\hline US & Texas & UTEP & H-8988 & 29.6101 & -098.9252 & GBIF \\
\hline US & Texas & UTEP & H-12339 & 31.4535 & -106.0993 & GBIF \\
\hline US & Texas & UTEP & $\mathrm{H}-18937$ & 33.0556 & -099.1784 & GBIF \\
\hline US & Texas & iNaturalist & 2543455 & 26.5964 & -097.5575 & GBIF \\
\hline US & Texas & iNaturalist & 250562 & 31.8621 & -098.9309 & GBIF \\
\hline US & Texas & iNaturalist & 293553 & 29.6218 & -103.0398 & GBIF \\
\hline US & Texas & iNaturalist & 301501 & 27.1379 & -098.3742 & GBIF \\
\hline US & Texas & iNaturalist & 301475 & 28.4718 & -098.3382 & GBIF \\
\hline
\end{tabular}


Table A1. Continued.

\begin{tabular}{|c|c|c|c|c|c|c|}
\hline Country & State & Collection & Catalog Number & Latitude & Longitude & Source \\
\hline US & Texas & iNaturalist & 717136 & 30.2264 & -103.6689 & GBIF \\
\hline US & Texas & ¡Naturalist & 1067867 & 26.285 & -097.4257 & GBIF \\
\hline US & Texas & iNaturalist & 1432285 & 27.6447 & -098.8395 & GBIF \\
\hline US & Texas & iNaturalist & 136359 & 32.7363 & -100.572 & GBIF \\
\hline US & Texas & ¡Naturalist & 1379956 & 28.1937 & -096.992 & GBIF \\
\hline US & Texas & iNaturalist & 1477039 & 29.1134 & -098.3701 & GBIF \\
\hline US & Texas & iNaturalist & 149101 & 37.8175 & -096.17 & GBIF \\
\hline US & Texas & iNaturalist & 1556139 & 31.1975 & -099.4938 & GBIF \\
\hline US & Texas & iNaturalist & 1503367 & 27.7352 & -098.8364 & GBIF \\
\hline US & Texas & iNaturalist & 1503372 & 27.465 & -098.8376 & GBIF \\
\hline US & Texas & iNaturalist & 1561359 & 27.4459 & -098.8368 & GBIF \\
\hline US & Texas & iNaturalist & 1610067 & 30.1978 & -102.8995 & GBIF \\
\hline US & Texas & ¡Naturalist & 1630260 & 30.0157 & -104.2101 & GBIF \\
\hline US & Texas & iNaturalist & 1728628 & 30.0953 & -097.926 & GBIF \\
\hline US & Texas & TCWC & 92297 & 31.1644 & -101.1803 & GBIF \\
\hline US & Texas & TCWC & 14272 & 28.1269 & -097.4317 & GBIF \\
\hline US & Texas & TCWC & 25976 & 31.4852 & -106.1386 & GBIF \\
\hline US & Texas & TCWC & 33623 & 26.2072 & -098.2294 & GBIF \\
\hline US & Texas & TCWC & 89850 & 32.1056 & -100.7139 & GBIF \\
\hline US & Texas & iNaturalist & 1137548 & 27.6671 & -098.8369 & GBIF \\
\hline US & Texas & TCWC & 10559 & 28.4606 & -098.2318 & GBIF \\
\hline US & Texas & TCWC & 18314 & 26.1982 & -098.0696 & GBIF \\
\hline US & Texas & UTEP & H-19721 & 30.7083 & -104.1923 & GBIF \\
\hline US & Texas & TCWC & 92920 & 27.4539 & -098.9169 & GBIF \\
\hline US & Texas & TCWC & 5193 & 32.2207 & -098.2364 & GBIF \\
\hline US & Texas & TCWC & 64220 & 31.7793 & -098.1951 & GBIF \\
\hline US & Texas & TCWC & 80499 & 32.7167 & -100.9177 & GBIF \\
\hline US & Texas & UBCBBM & H001156 & 28.0638 & -097.5092 & GBIF \\
\hline US & Texas & UBCBBM & H001165 & 27.5861 & -097.9348 & GBIF \\
\hline US & Texas & NCSM & 8764 & 32.3942 & -099.3947 & GBIF \\
\hline US & Texas & UTEP & $\mathrm{H}-18200$ & 31.9364 & -104.7188 & GBIF \\
\hline US & Texas & iNaturalist & 1620870 & 30.2346 & -103.3697 & GBIF \\
\hline US & Texas & iNaturalist & 1591618 & 30.1954 & -102.8929 & GBIF \\
\hline US & Texas & iNaturalist & 1391887 & 28.2416 & -098.5625 & GBIF \\
\hline US & Texas & iNaturalist & 1837850 & 30.039 & -097.9489 & GBIF \\
\hline US & Texas & iNaturalist & 1843096 & 30.8161 & -103.8642 & GBIF \\
\hline US & Texas & iNaturalist & 215970 & 26.5149 & -097.4859 & GBIF \\
\hline US & Texas & iNaturalist & 2082973 & 26.348 & -098.1828 & GBIF \\
\hline US & Texas & iNaturalist & 2506161 & 25.9305 & -097.3662 & GBIF \\
\hline US & Texas & ¡Naturalist & 2645138 & 29.3854 & -099.985 & GBIF \\
\hline US & Texas & iNaturalist & 300910 & 32.6283 & -101.3907 & GBIF \\
\hline US & Texas & iNaturalist & 758598 & 28.3056 & -096.9433 & GBIF \\
\hline US & Texas & iNaturalist & 812348 & 28.1218 & -097.4429 & GBIF \\
\hline US & Texas & iNaturalist & 760453 & 29.7065 & -101.2481 & GBIF \\
\hline US & Texas & iNaturalist & 1710062 & 26.8521 & -098.8893 & GBIF \\
\hline US & Texas & iNaturalist & 1629901 & 29.8424 & -103.212 & GBIF \\
\hline US & Texas & iNaturalist & 1629669 & 30.1193 & -103.2534 & GBIF \\
\hline US & Texas & iNaturalist & 1747565 & 29.7767 & -099.3863 & GBIF \\
\hline US & Texas & iNaturalist & 1265769 & 30.083 & -098.4134 & GBIF \\
\hline US & Texas & TCWC & 92938 & 30.0667 & -099.7103 & GBIF \\
\hline US & Texas & TCWC & 15035 & 30.2706 & -097.7453 & GBIF \\
\hline US & Texas & TCWC & 42862 & 29.455 & -098.6339 & GBIF \\
\hline US & Texas & TCWC & 52849 & 26.1212 & -097.9927 & GBIF \\
\hline US & Texas & TCWC & 77166 & 33.6442 & -098.8175 & GBIF \\
\hline US & Texas & TCWC & 77177 & 34.1306 & -098.1017 & GBIF \\
\hline US & Texas & TCWC & 77179 & 33.8128 & -098.1478 & GBIF \\
\hline US & Texas & TCWC & 77187 & 33.4131 & -098.8486 & GBIF \\
\hline US & Texas & TCWC & 79252 & 26.7596 & -098.842 & GBIF \\
\hline US & Texas & TCWC & 84340 & 33.4785 & -098.6688 & GBIF \\
\hline US & Texas & UBCBBM & H001145 & 28.0959 & -097.4636 & GBIF \\
\hline US & Texas & UTEP & H-15853 & 31.4792 & -103.5263 & GBIF \\
\hline
\end{tabular}


Table A1. Continued.

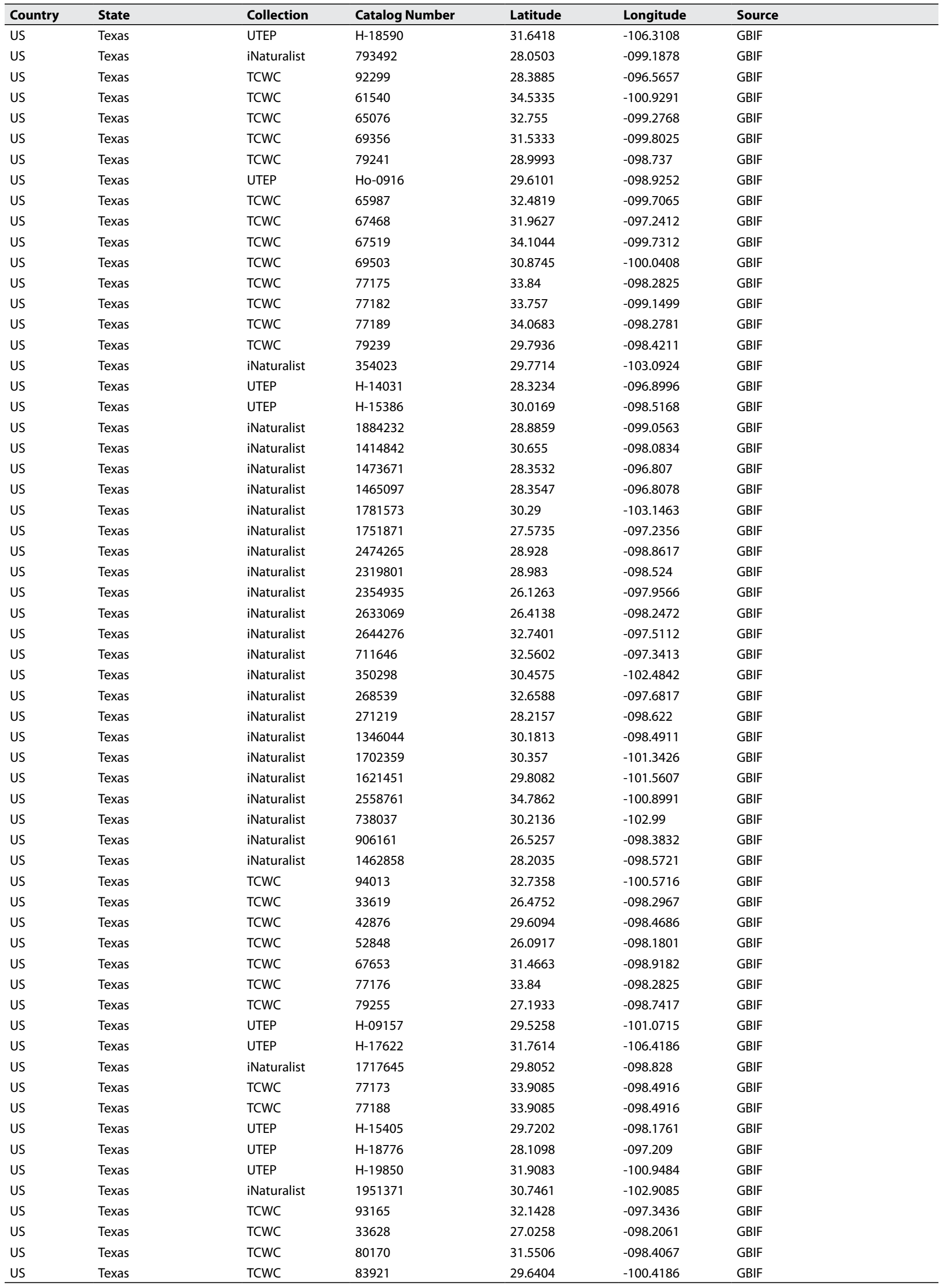


Table A1. Continued.

\begin{tabular}{|c|c|c|c|c|c|c|}
\hline Country & State & Collection & Catalog Number & Latitude & Longitude & Source \\
\hline US & Texas & UTEP & $\mathrm{H}-18676$ & 30.7495 & -098.1629 & GBIF \\
\hline US & Texas & UTEP & H-18531 & 28.2375 & -098.5633 & GBIF \\
\hline US & Texas & iNaturalist & 2356016 & 26.1264 & -097.9567 & GBIF \\
\hline US & Texas & iNaturalist & 1974657 & 29.4305 & -098.8632 & GBIF \\
\hline US & Texas & iNaturalist & 2397818 & 28.983 & -098.524 & GBIF \\
\hline US & Texas & iNaturalist & 247109 & 27.6613 & -098.8372 & GBIF \\
\hline US & Texas & iNaturalist & 2612484 & 26.4087 & -098.243 & GBIF \\
\hline US & Texas & iNaturalist & 257186 & 30.1454 & -102.3909 & GBIF \\
\hline US & Texas & iNaturalist & 2692785 & 26.4079 & -098.2378 & GBIF \\
\hline US & Texas & iNaturalist & 312157 & 27.7594 & -098.8359 & GBIF \\
\hline US & Texas & iNaturalist & 501677 & 29.8656 & -098.6423 & GBIF \\
\hline US & Texas & iNaturalist & 600951 & 32.651 & -098.5788 & GBIF \\
\hline US & Texas & iNaturalist & 722435 & 27.8735 & -097.7316 & GBIF \\
\hline US & Texas & iNaturalist & 760482 & 30.1445 & -102.4057 & GBIF \\
\hline US & Texas & iNaturalist & 806938 & 30.871 & -101.6968 & GBIF \\
\hline US & Texas & iNaturalist & 1765795 & 31.0376 & -100.5322 & GBIF \\
\hline US & Texas & iNaturalist & 1765709 & 30.2291 & -099.7197 & GBIF \\
\hline US & Texas & iNaturalist & 1765805 & 30.2036 & -102.9562 & GBIF \\
\hline US & Texas & iNaturalist & 136376 & 28.5401 & -099.4444 & GBIF \\
\hline US & Texas & iNaturalist & 1464486 & 29.9813 & -097.6288 & GBIF \\
\hline US & Texas & iNaturalist & 1467108 & 32.413 & -097.9601 & GBIF \\
\hline US & Texas & iNaturalist & 1541328 & 26.7259 & -098.8463 & GBIF \\
\hline US & Texas & iNaturalist & 1528494 & 27.0775 & -098.7945 & GBIF \\
\hline US & Texas & iNaturalist & 1591798 & 33.8891 & -099.4022 & GBIF \\
\hline US & Texas & iNaturalist & 1594880 & 28.2708 & -096.8258 & GBIF \\
\hline US & Texas & iNaturalist & 1617861 & 30.1996 & -102.0482 & GBIF \\
\hline US & Texas & iNaturalist & 1702932 & 30.5318 & -101.1618 & GBIF \\
\hline US & Texas & iNaturalist & 1601757 & 31.7614 & -106.4178 & GBIF \\
\hline US & Texas & iNaturalist & 1620709 & 30.4684 & -102.4158 & GBIF \\
\hline US & Texas & iNaturalist & 796058 & 29.0515 & -098.5883 & GBIF \\
\hline US & Texas & iNaturalist & 1128697 & 30.2021 & -102.9181 & GBIF \\
\hline US & Texas & TCWC & 23468 & 31.7267 & -099.1981 & GBIF \\
\hline US & Texas & TCWC & 43779 & 32.8288 & -098.6213 & GBIF \\
\hline US & Texas & TCWC & 77169 & 34.2435 & -099.9768 & GBIF \\
\hline US & Texas & TCWC & 89390 & 29.1047 & -098.1797 & GBIF \\
\hline US & Texas & TCWC & 89772 & 28.163 & -097.0189 & GBIF \\
\hline US & Texas & CAS & 10403 & 34.8663 & -100.9686 & GBIF \\
\hline US & Texas & UBCBBM & H001228 & 28.1071 & -097.368 & GBIF \\
\hline US & Texas & UTEP & $\mathrm{H}-6119$ & 30.9319 & -103.7917 & GBIF \\
\hline US & Texas & UTEP & $\mathrm{H}-16270$ & 35.7352 & -100.5746 & GBIF \\
\hline US & Texas & TCWC & 94777 & 31.2864 & -102.1318 & GBIF \\
\hline US & Texas & TCWC & 91945 & 30.3181 & -103.7711 & GBIF \\
\hline US & Texas & TCWC & 92939 & 29.9486 & -100.1733 & GBIF \\
\hline US & Texas & TCWC & 94012 & 32.7358 & -100.5716 & GBIF \\
\hline US & Texas & TCWC & 5194 & 32.1286 & -098.0935 & GBIF \\
\hline US & Texas & TCWC & 48068 & 31.9274 & -097.6491 & GBIF \\
\hline US & Texas & TCWC & 67073 & 31.5378 & -099.7094 & GBIF \\
\hline US & Texas & TCWC & 79253 & 27.3479 & -098.6161 & GBIF \\
\hline US & Texas & UTEP & $\mathrm{H}-18414$ & 36.2278 & -100.6502 & GBIF \\
\hline US & Texas & CAS & 22426 & 32.6751 & -097.4679 & GBIF \\
\hline US & Texas & TCWC & 92438 & 28.4242 & -096.5434 & GBIF \\
\hline US & Texas & TCWC & 77167 & 33.7011 & -098.8077 & GBIF \\
\hline US & Texas & TCWC & 77193 & 34.0652 & -098.6094 & GBIF \\
\hline US & Texas & TCWC & 79243 & 26.0868 & -097.9686 & GBIF \\
\hline US & Texas & TCWC & 90152 & 30.1239 & -103.5803 & GBIF \\
\hline US & Texas & UBCBBM & H001166 & 28.1071 & -097.368 & GBIF \\
\hline US & Texas & UBCBBM & H001206 & 28.0959 & -097.4636 & GBIF \\
\hline US & Texas & UBCBBM & H001230 & 27.9325 & -097.5822 & GBIF \\
\hline US & Texas & UTEP & H-17668 & 29.2052 & -099.8995 & GBIF \\
\hline US & Texas & iNaturalist & 1079099 & 26.3484 & -098.234 & GBIF \\
\hline US & Texas & iNaturalist & 1236311 & 26.4656 & -098.2213 & GBIF \\
\hline
\end{tabular}


Table A1. Continued.

\begin{tabular}{|c|c|c|c|c|c|c|}
\hline Country & State & Collection & Catalog Number & Latitude & Longitude & Source \\
\hline US & Texas & iNaturalist & 1339928 & 28.1691 & -098.6775 & GBIF \\
\hline US & Texas & iNaturalist & 1243169 & 27.6403 & -097.3919 & GBIF \\
\hline US & Texas & iNaturalist & 1391886 & 28.1434 & -098.6169 & GBIF \\
\hline US & Texas & iNaturalist & 1494583 & 30.3478 & -097.2934 & GBIF \\
\hline US & Texas & iNaturalist & 1464514 & 29.9988 & -097.6063 & GBIF \\
\hline US & Texas & iNaturalist & 1472943 & 27.2439 & -098.9349 & GBIF \\
\hline US & Texas & iNaturalist & 1589138 & 28.9459 & -098.8606 & GBIF \\
\hline US & Texas & iNaturalist & 1599444 & 33.9205 & -098.7592 & GBIF \\
\hline US & Texas & iNaturalist & 1605795 & 30.1439 & -102.5965 & GBIF \\
\hline US & Texas & iNaturalist & 1652289 & 31.2271 & -099.288 & GBIF \\
\hline US & Texas & iNaturalist & 1781583 & 30.4234 & -102.9706 & GBIF \\
\hline US & Texas & iNaturalist & 1662353 & 29.6602 & -101.149 & GBIF \\
\hline US & Texas & iNaturalist & 1695318 & 31.8944 & -102.3007 & GBIF \\
\hline US & Texas & iNaturalist & 1849242 & 30.213 & -103.0627 & GBIF \\
\hline US & Texas & iNaturalist & 1903232 & 26.7594 & -098.6133 & GBIF \\
\hline US & Texas & iNaturalist & 1945629 & 26.4948 & -098.0166 & GBIF \\
\hline US & Texas & iNaturalist & 2371566 & 30.2081 & -098.4493 & GBIF \\
\hline US & Texas & iNaturalist & 2481749 & 26.4454 & -098.2414 & GBIF \\
\hline US & Texas & iNaturalist & 2474339 & 26.5146 & -098.611 & GBIF \\
\hline US & Texas & iNaturalist & 295680 & 28.4153 & -096.6665 & GBIF \\
\hline US & Texas & iNaturalist & 711924 & 29.8596 & -101.6991 & GBIF \\
\hline US & Texas & iNaturalist & 739401 & 30.0111 & -097.7638 & GBIF \\
\hline US & Texas & iNaturalist & 814367 & 33.8977 & -099.4418 & GBIF \\
\hline US & Utah & BYU & 18896 & 38.5733 & -109.5492 & GBIF \\
\hline US & Utah & UCM & AC-143 & 40.5081 & -109.1939 & GBIF \\
\hline
\end{tabular}

